\title{
Reconciliation in a Hug: On Discovery of a Father from Archetypal Approach
}

\author{
Hui Shi \\ Sichuan University, Chengdu 610000, China \\ Hubei University for Nationality, Enshi 445000, China \\ shihuixili@163.com
}

\begin{abstract}
Sherwood Anderson's Discovery of a Father tells a story of the relationship between father and son which changes from alienation to understanding. Through an elaborate epiphany, the boy releases his hatred and rediscovers his father's good nature. The exquisite device or usage of images such as mother as female Noah, Oedipus complex, water, darkness, pond, light, etc. in the story will be focused on to demonstrate the rationality and inevitability of the happy ending, and a study of the relationship between father and son is helpful for us to establish a harmonious family in modern time.
\end{abstract}

Keywords: female Noah; image; reconciliation; archetype.

\section{在拥抱中和解一从原型理论看《认识父亲》中父子关系}

\author{
施慧 \\ 四川大学, 成都 中国 \\ 湖北民族学院, 恩施 中国
}

摘 要: 美国现代作家舍伍德・安德森的短篇小说《认识父亲》描述了一对父子由疏远、冷 漠走向理解、认同。作者精心设计了一种精神上的顿悟, 并且通过小男孩这种瞬间灵光一闪 释放了其长期以来对父亲的厌恶情绪, 最终和父亲和解。这个过程看似突然, 却是和小说人 物塑造和细节安排密切相关。本文试图从母亲的形象、水、黑暗、池塘、灯、俄狄浦斯情节 等意象着手, 分析父子和解的合理性和必然性。以此为探讨怎样正确处理父子、母子关系, 建立和谐幸福家庭寻找有效方式。

关键词: 女诺亚; 意象; 父子和解; 原型批评.

\section{1. 前言}

美国现代作家舍伍德 - 安德森（Sherwood Anderson）（1876-1941）的代表作是《俄亥俄州的 温斯堡镇》(Winesburg, Ohio)。该小说由一篇题为 “畸人志” ( “The book of grotesques”) 的引言和二十四个独立又彼此关联的短篇聚集而成, 被誉为美国第一部真正意义上的现代小 说。作为美国现代小说的先声, 安德森把笔触深入到了人物的精神层面, 勾勒出了一群小镇居 民 “畸形” 的心理世界。批评家普遍认为他的小说中充满了现代人的孤独感、隔绝感、压抑 感和挫败感。而人们无法有效地表达自己的思想和情感是造成以上种种痛苦的根源。学者们 认为安德森的关注点历来就是人与人之间的沟通障碍问题。《认识父亲》 (Discovery of a Father）（选自《讲故事者的故事》）（A Story Teller's Story）是安德森一篇非常有代 表性的短篇小说。小说从一个小男孩的视角来叙述故事: 他以父亲常常做过的最最荒谬的事 情开头, 列举父亲的行为给他带来的重重难堪和痛苦。在他看来父亲游手好闲、不务正业、 爱交狐朋狗友, 最让他无法忍受的是父亲的胡说八道、信口乱掐。每次听到父亲的 “精彩讲 演” , 他就觉得父亲无聊、丢脸, 甚至时常希望他不是自己的亲生父亲。他时常为母亲难过, 
不能理解这样一位不负责任、胡吹海㑆的俊瓜总是能被她容忍甚至是欣赏。和父亲之间的冷 漠、疏远关系一直持续了数年, 但是突然在一个雨夜的小池塘里, 父亲和他共泳。两人之间 也只有零翏几句平常不过的对白。但正是在这样的闪电瞬间, 小男孩对父亲产生了莫名的情 感冲动, 雬时开始认识父亲, 并重新理解父亲的陌生和魅力。“这是第一次我认识到我是我 父亲的儿子。他是一位像我向往的那样讲故事的人。我可能在黑暗中微笑了一下。如果我真 的那么做了, 那一定是因为我明白了我永不盼望另一个父亲而笑的”。（Anderson， 2002, p. 35)

池塘里寻常的父子共泳的一幕似乎是日常生活中最平淡无奇的琐事, 却能让小男孩瞬间产生 精神顿悟。从整个故事的情节发展看, 似乎没有任何痕迹和暗示, 也不是父亲这个人物性格 发展的自然结果。同时, 这一幕发生在某一个雨夜, 在家附近一个无名小池塘里, 父子之间 也沉默居多, 却能让小男孩对父亲产生强烈的情感冲击。他开始撕破隔膜、重新理解父亲、 恢复和父亲应有的亲密关系。这样的情节安排似乎是飞来之笔, 是作者的故意安排, 看似不 甚自然。但如果从文章中一些特殊的意象如: 水、黑暗、池塘、灯、俄狄浦斯情节等细节处 审察, 也不难发现安德森对父子和解这个必然结局设计的合理性和精妙处。

\section{2. 神话原型理论}

从 20 世纪 30 年代开始, 西方文学批评界兴起了以诺斯洛普 - 弗莱（Northrop Frye）为代表 的神话批评流派。到 50 年代他又确立了原型批评的概念。在其《批评的解剖》 (Anatomy of Criticism）一书中, 他对原型定义为 “我给它取名为 “原型” (archetype), 也即是一种 典型的或反复出现的形象。我所说的原型, 是指将这一首诗与另一首诗联系起来的象征, 可 用以把我们的文学经验统一并整合起来。而且鉴于原型是可以供人们交流的象征、故原型批 评所关心的, 主要是要把文学视为一种社会现象、一种交流的模式”。(Frye, 2006, p. 142) 卡尔・荣格（Carl G. Jung ）是原型分析的另一重要代表人物。他对神话原型批评的贡献 主要在于对种族记忆和原型的提出。他认为人的意识有包含各种情节的个体潜意识, 也有体 现种种原型的集体无意识。集体无意识是我们祖先反复经验所形成的原始意象, 它以某种重 复或类似的方式对特定刺激作出反应。这些意象体现在神话、民间传说、宗教、幻想、童话、 梦和文学中。艺术家拥有超凡的想象力, 更善于用意象来表达人类共同的体验和感受。而借 助神话的形式, 则更能使其更合适的表达出来。可以说, 弗莱引导我们把原型应用到文学作 品分析中, 而荣格则把这种理论和心理学结合起来, 使之成为一种文学研究的途径或文学批 评方法。

\section{3. 母亲形象}

在《认识父亲》这篇小文中, 父亲是当之无愧的主角。安德森似乎想采用欲扬先抑的手法如 标题所示一样, 不断地以小见大, 发现父亲的长处、认识父亲的优点。在这个长期磨合的过 程中，母亲在男孩和父亲关系的调和中发挥了重大的作用。

在后来的 “发现” 之夜，母亲也是伴着屋外的雨在等待着父子的归来。雨水，池水，在男孩 思想顿悟的过程中似乎发挥了决定性的作用。水的出现总能让人联想起《圣经》中诺亚方舟 的故事。在这则故事中, 世界一片混乱, 原本和谐的社会失去了固有秩序, 上帝逐渐对人类 丧失信心, 决定消除这些罪恶。于是, 他让世间发起了一场大洪水。洪水来势汹汹, 世人如 临末日。洪水过后, 一切往日的混乱和邪恶都随着肆虐的洪水消失。所幸, 在这次大清扫中, 被上帝选中的纯洁、无辜的诺亚及其家人存活了下来。在洪水中, 他拯救了家人, 并带领他 们重建水灾后的新世界。因此, 在西方文学中, 水总能与毁灭和新生紧密联系。在《认识父 亲》中, 母亲似乎充当了诺亚的角色, 在父子长期存在隔阂、不理解甚至敌对的不和谐家庭 中, 她总是温柔地起着润滑的作用, 多次在外人面前赞扬丈夫, 在丈夫出糗时支持、爱护, 在儿子心中树立父亲正面形象。这个家庭关系不和谐的小家，终于在一个阴雨绵绵的夜间引 
来了转折。在这一天, 雨水中、池水中, 小男孩突然原谅了父亲、开始向父亲敞开内心, 并 最后重新理解父亲和自己的父子亲情。至此, 这个曾经不和美、不温馨的小家开始重建秩序, 走向新生。母亲的女诺亚形象不言而喻, 在这场父子关系由失控到重归和谐的 “洪灾” 中, 她的作用至关重要。

\section{4. 父子和解}

在这场看似突兀的父子由长期隔膜、抗拒到突然地敞开心扉、取得和解的反转剧中，母亲的 介入十分关键。安德森还设计了其他的细节来铺成男孩重新认识父亲的基础。如: 俄狄浦斯 情节的应用。在《认识父亲》中，男孩对父亲的厌恶、不理解似乎是与生俱来的。弗洛伊德 通过希腊神话中俄狄浦斯的故事，即儿子在不知情的情况下杀父娶母的故事来印证男孩潜意 识中对父亲有一种惧怕和对抗。在小说中，男孩很明显具有这种倾向，他被母亲吸引、热爱 母亲, 觉得父亲浮夸、爱撒谎、说大话、不务正业, 不能容忍母亲为这样不负责任的男人辩 护，更为母亲这段 “不幸” 的婚姻抱不平。这里可以说安德森似乎想叙述一个美国式的俄狄 浦斯的故事。但是不同于原作，安德森给了男孩突破母亲吸引而认同父亲的机会。这个让男 孩健全人格的机会是他在无意识中得到的。

这个决胜的时刻被安德森有意地安排在一个下雨的夜晚。外面一片黑暗，父子之间几乎没有 交流, 甚至在池塘里游泳两人也保持沉默。其中有个细节, 父亲和男孩都是脱光了进入池塘。 在此之前男孩有抗拒、不愿意脱衣服。父亲要求他脱光。这样，安德森就把这对父子的 “认 识”之夜设置在一个阴雨绵绵的夜间, 父子两人在家附近的池塘里裸泳, 然后一起归家。母 亲在一盏小灯下微笑地等待父子归来。于是, 男孩觉得已经能理解父亲, 摆脱对他的敌意并 以他为荣。根据弗洛伊德和荣格的理论, 黑暗的夜就是一种神秘、混沌。池塘等能当做容器 的东西都被看做是女性的象征。池塘里有水, 父子两人脱掉成见的外衣在一片混沌中进入荡 漾着雨水的池塘中, 裸袿相见。这一场景完全可以看做幼儿在母亲子宫里活动的样子。两个 人在一个神圣、纯洁的地方, 都进入了一个无意识的层面。男孩天性中对强者的渴望涌现出 来，依靠自己的本能向父亲靠近。回到家时，灯光下的母亲的形象更像一位天使一样，微笑 着呵护着这初生的理解和仰慕。

\section{5. 结论}

安德森的《发现父亲》给我们讲述了一个 20 世纪美国版的俄狄浦斯故事，但这个故事的结局 是属于安德森式的结尾。在母亲的调和作用下, 在一个特定的场合中, 男孩进入了一种无意 识的状态，激发出了对父亲亲近的本能，最终摒弃了对父亲的成见，重新认识父亲。这则温 情的小故事在作者的精心设计下, 娓娓动听, 激起读者对家庭重要性的思考, 对和谐父子(女)、 母子（女）亲密关系的向往与期许。虽然距离安德森的时代几乎过去了一百年，但这种温暖、 和谐的家庭关系仍然是社会建设的重心。

\section{References}

[1]. Anderson, Sherwood. "Discovery of a Father" in Contemporary College English Intensive Reading 3. Beijing: Foreign Language Teaching and Research Press, 2002, pp.32-35.

[2]. Crowley, Malcolm. Introduction to Winesburg, Ohio. New York: The Viking Press, Inc., 1960, p.1.

[3]. Frye, Northrop. The Anatomy of Criticism, Chen Hui, trans. Tianjin: Baihua Literature and Art Publishing House, 2006.

[4]. Jung, Karl. Make Us Return to Our Spiritual Home, Feng Chuan, trans. Beijing: Reform Press, 1997. 
[5]. Jung, Karl. The Archetypes and the Collective Unscious, Xu Delin, trans. Beijing: International Culture Press Limited, 2011. 